\title{
A TP53 mutation model for the prediction of prognosis and therapeutic responses in head and neck squamous cell carcinoma
}

\author{
Congyu Shi', Shan Liu', Xudong Tian' ${ }^{1}$ Xiaoyi Wang ${ }^{1}$ and Pan Gao ${ }^{2 *}$
}

\begin{abstract}
Background: Tumor protein p53 (TP53) is the most frequently mutated gene in head and neck squamous cell carcinoma (HNSC), and TP53 mutations are associated with inhibited immune signatures and poor prognosis. We established a TP53 mutation associated risk score model to evaluate the prognosis and therapeutic responses of patients with HNSC.
\end{abstract}

Methods: Differentially expressed genes between patients with and without TP53 mutations were determined by using data from the HNSC cohort in The Cancer Genome Atlas database. Patients with HNSC were divided into high- and low-risk groups based on a prognostic risk score that was generated from ten TP53 mutation associated genes via the multivariate Cox regression model.

Results: TP53 was the most common mutant gene in HNSC, and TP53 mutations were associated with immunogenic signatures, including the infiltration of immune cells and expression of immune-associated genes. Patients in the high-risk group had significantly poorer overall survival than those in the low-risk group. The highrisk group showed less response to anti-programmed cell death protein 1 (PD-1) therapy but high sensitivity to some chemotherapies.

Conclusion: The risk score based on our TP53 mutation model was associated with poorer survival and could act as a specific predictor for assessing prognosis and therapeutic response in patients with HNSC.

Keywords: TP53 mutation, Head and neck squamous cell carcinoma, Prognosis, Immunotherapy, Chemotherapy, Therapeutic response

\section{Introduction}

Head and neck cancers mainly refer to a group of malignancies that originate from the moist mucosal surfaces lining the oral cavity, pharynx (including nasopharynx, oropharynx, and hypopharynx), larynx, and paranasal sinuses and nasal cavity. Head and neck squamous cell carcinoma (HNSC) begins in the squamous cells of mucosa and accounts for more than $90 \%$ of all head and

\footnotetext{
* Correspondence: gaopan@scu.edu.cn

${ }^{2}$ State Key Laboratory of Oral Diseases \& National Clinical Research Center for Oral Diseases \& Department of General and Emergency Dentistry, West China Hospital of Stomatology, Sichuan University, Chengdu, China Full list of author information is available at the end of the article
}

neck cancers and is associated with high lethality [1, 2]. HNSC is estimated to cause over 600,000 new cases and 380,000 deaths annually worldwide [3, 4]. The consumption of tobacco and alcohol contributes to nearly $75 \%$ of HNSC morbidity [5]. Additionally, human papillomavirus (HPV) has been proven to be a vital risk factor for a subset of HNSC, oropharyngeal squamous cell carcinoma (OPSC) [6]. Evidence shows that HPV status is related to the prognosis of patients with HNSC. $\mathrm{HPV}^{+}$ HNSC, particularly $\mathrm{HPV}^{+}$OPSC, is associated with a more favorable prognosis than $\mathrm{HPV}^{-} \mathrm{HNSC}$, although the effect of HPV outside the oropharynx is still unclear

C C The Author(s). 2021 Open Access This article is licensed under a Creative Commons Attribution 4.0 International License, which permits use, sharing, adaptation, distribution and reproduction in any medium or format, as long as you give appropriate credit to the original author(s) and the source, provide a link to the Creative Commons licence, and indicate if changes were made. The images or other third party material in this article are included in the article's Creative Commons licence, unless indicated otherwise in a credit line to the material. If material is not included in the article's Creative Commons licence and your intended use is not permitted by statutory regulation or exceeds the permitted use, you will need to obtain permission directly from the copyright holder. To view a copy of this licence, visit http://creativecommons.org/licenses/by/4.0/ The Creative Commons Public Domain Dedication waiver (http://creativecommons.org/publicdomain/zero/1.0/) applies to the data made available in this article, unless otherwise stated in a credit line to the data. 
[7]. Consequently, the American Joint Committee on Cancer (AJCC) downgraded the stage of patients with $\mathrm{HPV}^{+}$OPSC in its eighth-edition staging manual [8]. However, the optimal therapeutic methods should be determined based on more rigorous trials of definitive therapy [9]. As a result, authentication of predictive biomarkers will be beneficial to the evaluation of prognosis and surveillance of therapeutic response on $\mathrm{HPV}^{+/-}$in HNSC.

Tumor protein p53 (TP53), encoding protein p53, is the most frequently mutated gene in HNSC [10], but the mutation is absent in the $\mathrm{HPV}^{+}$subgroup [11]. Functioning as a tumor suppressor gene (TSG), TP53 regulates multiple biological processes, including DNA repair, cell cycle arrest, apoptosis, senescence, maintenance of stem cells, and angiogenesis [12]. The loss of wildtype TP53 or gain of oncogenic TP53 function, predominately caused by missense mutations, is associated with inhibited immune signatures [13], poorer prognosis and resistance to radio-/chemotherapy [14], which makes the status of TP53 to be an effective predictive biomarker to evaluate prognosis and surveillance to therapeutic responses in HNSC. Given the tumor heterogeneity in the immune microenvironment of HNSC, we hypothesized that some immune-associated signatures influenced by TP53 mutations might play crucial roles in assessing prognosis and therapeutic responses and even guiding clinical treatment for HNSC. Thus, in our current study, we established a TP53 mutationrelated risk score model, which may affect the therapeutic response of HNSC patients.

\section{Material and methods}

Identification of differentially expressed genes (DEGs) and gene set enrichment analysis (GSEA)

The RNA-seq data (raw counts) from The Cancer Genome Atlas (TCGA) were normalized using the voom function from the "limma" package, and DEGs between patients with $(n=340)$ and without $(n=160)$ TP53 mutations in the TCGA HNSC cohort were acquired by "limma" in the standard comparison mode [15]. The DEG threshold was set at $\mid \log 2$-fold change $\mid \geq 1$ and $P$ value adjusted $<0.05$. KEGG GSEA annotation of DEGs was performed using the $\mathrm{R}$ package "clusterProfiler" [16-19]. The GSEA enrichment threshold was set at $P$ value $<0.05$, FDR q value $<0.25$ and $|\mathrm{NES}|>1.0[20,21]$. TP53 was re-annotated based on the results of the HNSC mutec maf file downloaded by TCGA-GDC and intersected with the tumor transcriptome. The tumor samples were divided into groups of wildtype and mutate-benign (including that the IMPACT result was LOW, as well as the IMPACT result is LOW and the PolyPhen results were benign) and mutate-non-benign according to the status of TP53. Their sample counts are 160 and 27 and 313 respectively.

\section{Construction of the TP53-associated prognostic model}

Initially, univariate Cox analysis with $P<0.001$ was used to evaluate the relationship between DEGs and the survival time of HNSC patients [22]. To further narrow the scope of the candidate prognostic DEGs, we adopted the multivariate Cox regression and step backward Cox regression after primary filtration [23]. The linear combination of the regression coefficient $(\beta)$ derived from the multivariate Cox regression model multiplied by its mRNA level generated a prognostic risk score with ten genes. The risk score for each patient was calculated based on the risk score formula: Risk Score $=$ expression of gene $1 \times \beta 1+$ expression of gene $2 \times \beta 2+\ldots$ expression of gene $n \times \beta n$ [24]:

$$
\text { Risk Score }=\sum_{i=1}^{n}\left(\text { Expression }_{i} \times \beta i\right)
$$

After that, the patients were divided into high- and low-risk groups by setting the median value of risk scores as cutoff value. The overall survival (OS) of these two groups was calculated by the Kaplan-Meier method with the log-rank test. Receiver operating characteristic (ROC) curves were generated to assess the predictive performance of the prognostic model [25]. The expression patterns of DEGs in this prognostic model were visualized by the "pheatmap" package (https://cran.rproject.org/web/packages/pheatmap/index.html).

\section{Validation of the prognostic model in external dataset}

The predictive performance of the gene signature model was further validated in GSE65858. Samples with an overall survival time of less than 60 days were filtered, and then they were divided into high- or low-risk groups according to the formula of the risk score derived from the training dataset. Kaplan-Meier (KM) survival analysis and receiver operating characteristic (ROC) curves were used to evaluate the predictive power of the gene signature, and the prognostic performance of other clinicopathological factors was also analyzed. TP53 mutational status was not available in this cohort.

\section{Evaluation of the immune microenvironment between the high-risk and low-risk groups}

We used the CIBERSORT algorithm to analyze the fractions of 22 immune cell types using 1000 permutations and the LM22 gene signature of TCGA-HNSC samples in the $\mathrm{R}$ language as recommended by the authors [26]. The result was subsequently analyzed. 


\section{Immunotherapeutic and chemotherapeutic response prediction}

In recent years, immune checkpoint inhibitors have been proven to be a promising therapy for various cancers. Inhibitors targeting programmed cell death protein 1 ( $P D$ 1) and CTLA-4 may enhance antitumor immunity, while tumor immune escape restricts the application of immune checkpoint blockade. The computational method of Tumor Immune Dysfunction and Exclusion (TIDE) modeled immune escape in tumors with distinct levels of cytotoxic $\mathrm{T}$ lymphocytes by using both $\mathrm{T}$ cell dysfunction and exclusion signatures [27]. In this current study, TIDE was used to predict clinical responses of HNSC to immune checkpoint inhibitors by website tools (http:// tide.dfci.harvard.edu). Chemotherapy is an important method to treat HNSC, therefore the "pRRophetic" package was employed for the prediction of chemotherapeutic response, quantified by the half maximal inhibitory concentration (IC50) of each HNSC patient [28, 29]. These results were compared between the two risk groups by Kruskal-Wallis test or Chi squared test.

\section{Independence of the gene-related prognostic signature from other clinical features}

Univariate and multivariate Cox regression analyses were conducted to determine whether the risk score calculated from the prognostic model was an independent prognostic factor for HNSC patients after considering other clinical features, including age, sex and AJCC stage, and nomograms were constructed to assess the probability of 1-, 2-, and 3-year overall survival for HNSC patients based on the signature [30].

\section{Results}

\section{Identification of DEGs between HNSC patients with and} without TP53 mutations

We identified mutations using data from the HNSC cohort in TCGA and found that TP53 was the most frequently mutated gene, occurring in $63 \%$ of cases, and its mutations were mutually exclusive with HPV presence in HNSC. The difference in clinical characteristics and immune cell infiltration between $\mathrm{HPV}^{-}$and $\mathrm{HPV}^{+}$ HNSC were analyzed. Tumor inhibition associated immune cells, including CD8 $\mathrm{T}$ cells, CD4 memory activated $\mathrm{T}$ cells, and follicular helper $\mathrm{T}$ cells, were more abundant in $\mathrm{HPV}^{+} \mathrm{HNSC}$. And the tumor promotion associated immune cells, including Macrophages M2, CD4 memory resting $\mathrm{T}$ cells, and Macrophages $\mathrm{M} 0$, were more abundant in $\mathrm{HPV}^{-}$HNSC (Supplementary Tables 1 and 2). The majority of TP53 mutations were missense mutations, followed by nonsense mutations and frameshift deletions (Fig. 1A). In this study, we divided patients with HNSC into TP53-mutated and wildtype groups and investigated the corresponding DEGs. In total, 508 upregulated genes and 197 downregulated genes were discerned. To elucidate the functions of DEGs, GSEA was carried out. As shown in Fig. 1B, immune activated signals, including the $\mathrm{T}$ cell receptor signaling pathway, natural killer cell mediated cytotoxicity, primary immunodeficiency, and antigen processing and presentation, were enriched in TP53 wildtype HNSC tumor tissues, which indicated that the TP53 mutation status may influence the immune response to HNSC. In the re-analysis, 35 mutate-benign were eliminated for differential expression analysis. The results showed that the 10 genes in the risk score model were still in the significant DEGs (Supplementary Figure 1A), which were shown in the volcano map. We supplemented the Kaplan-Meier analysis according to the annotation results. There was no significant difference in overall survival between mutate-benign group and mutate-nonbenign group $(P>0.05$; Supplementary Figure 1B). Compared with wildtype group, the group of mutate-nonbenign had poorer prognosis $(P<0.05$; Supplementary Figure $1 \mathrm{~B})$. The overall survival between groups divided by the result of PolyPhen annotation was not statistically significant (Supplementary Figure 1C).

\section{Construction of the prognostic signature model based on DEGs}

Thirty genes with $P<0.001$ were left after the univariate Cox analysis of DEGs (508 upregulated DEGs + 197 downregulated DEGs). More information of the 30 genes were displayed in Supplementary Table S3. Then we adopted backward stepwise multivariate Cox regression analysis of the 30 genes, ten genes were identified to build the prognostic model. A risk score based on ten genes for predicting prognostic value was calculated based on the formula: RiskScore $=$ WNT7A * $0.275+$ TMSB4Y* $(-0.541)+$ SPINK6 * $(-0.196)+$ ZNF831* ($3.2)+$ GZMM * $(-0.417)+$ FDCSP * $(-0.058)+$ SH2D1A * $0.584+D K K 1 * 0.102+C H G B * 0.131+I K Z F 3 * 0.629$. The basic information of the ten genes was shown in Supplementary Table 4. Most of the ten genes are associated with cancer and immune signaling pathways. WNT7A, SPINK6, DKK1, and CHGB were upregulated, and the rest of the ten genes were downregulated in TP53-mutated group in comparison with TP53-wildtype group (Supplementary Figure 2). The vital status and heatmap of prognostic genes are presented (Fig. 2A). Kaplan-Meier survival curves showed that patients classified in the high-risk group were more likely to have significantly worse overall survival than those classified in the low-risk group $(P<0.0001$; Fig. $2 \mathrm{~B})$. The ROC curves and corresponding area under the curve (AUC) showed that the model based on the risk score was better than the model based on clinical characteristics, including age, sex, grade and stage (Fig. 2C). 


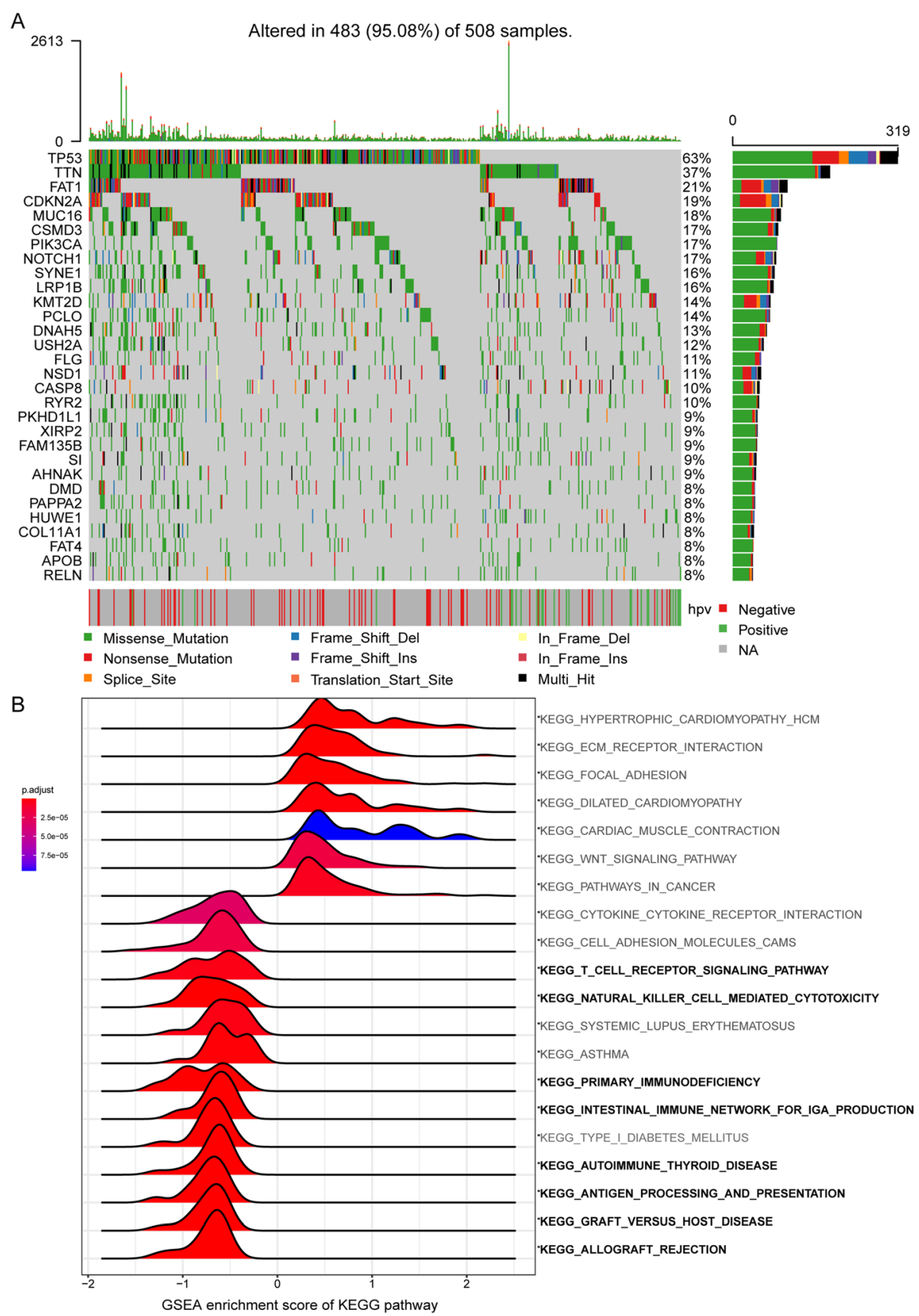

Fig. 1 TP53 mutation-associated genes in the TCGA HNSC cohort and GSEA of KEGG pathways. A The mutated genes were listed according to their mutation frequencies, considering the status of HPV infection. The mutations include missense mutations, nonsense mutations, splice-site mutations, frameshift mutations, and in-frame mutations. The oncoplot were drawn in maftools package. B Gene set enrichment analysis (GSEA) was performed to enrich the KEGG pathways in genes that were related to TP53 mutation. Significant enrichments in immune-related KEGG pathways are labeled in bold. A false discovery rate (FDR) of less than 0.05 and an absolute value of the enrichment score (ES) of greater than 0.5 were defined as the cutoff criteria. The analysis were finished using clusterProfiler package

\section{External validation of the prognostic signature model based on DEGs}

The GSE65858 cohort of HNSC, a dataset from the Gene Expression Omnibus (GEO), was analyzed using the constructed model. The risk score based on ten genes for evaluating prognostic value was calculated, and the vital status and heatmap of prognostic genes were indicated (Fig. 3A). Kaplan-Meier survival analysis showed that patients grouped into the high-risk set tended to have poorer overall survival than those in the 

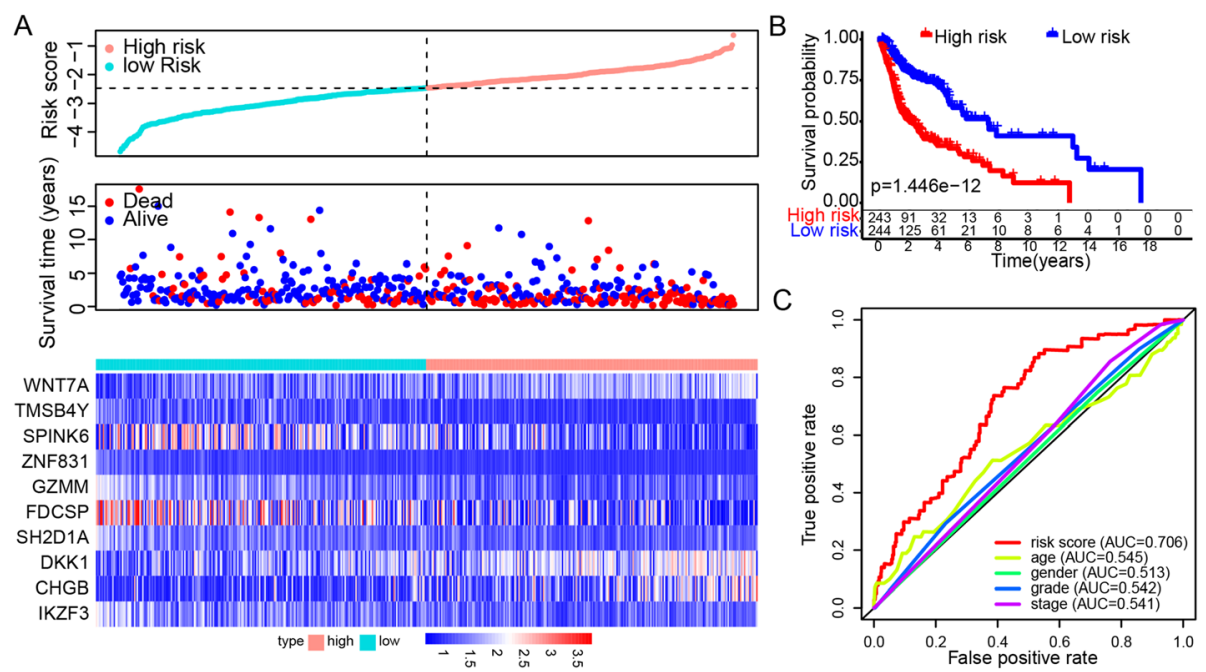

Fig. 2 Construction of the DEG-based gene signature prognostic model. A Risk score, vital status and heatmap of prognostic genes in the highand low-risk groups. B Kaplan-Meier survival curves of the relative overall survival of high- and low-risk patients. C The ROC curve for one-year survival of the gene signature and clinical features. DEGs were identified between TP53 mutated and wildtype TCGA-HNSC samples (Padj < 0.05 , $|\log F C|>1)$. Prognostic DEGs were screened with coxph $P$ value $<0.001$, these genes were displayed in Supplementary Table 3 . The risk model was constructed after stepwise coxph analysis with survival package. The information of ten genes in model were summarized in supplementary Table 4

low-risk group $(P<0.05$; Fig. 3B). The ROC curves and corresponding AUC showed that the model based on risk score was better than clinic features, including age, sex, grade and stage (Fig. 3C).

\section{Kaplan-Meier analyses of overall survival according to TP53 mutation status}

Consistent with a previous report [31], our study found that TP53 mutation status was associated with prognosis; namely, HNSC patients with TP53 mutation had poorer prognosis $(P<0.05$; Fig. $4 \mathrm{~A})$. To elucidate whether the risk score is independent of TP53 mutation, patients with HNSC were grouped into distinct sets according to TP53 mutation or wildtype with high- or low-risk status (Fig. 4B). The patients with both a highrisk score and TP53 mutations were inclined to have the worst overall survival in comparison with any other single or combined factors $(P<0.0001$; Fig. 4B). However, the status of HPV was not associated with the prognosis of HNSC (Supplementary Figure 3A). The risk score was also independent of the HPV status (Supplementary Figure 3B-D). Kaplan-Meier overall survival curves of the
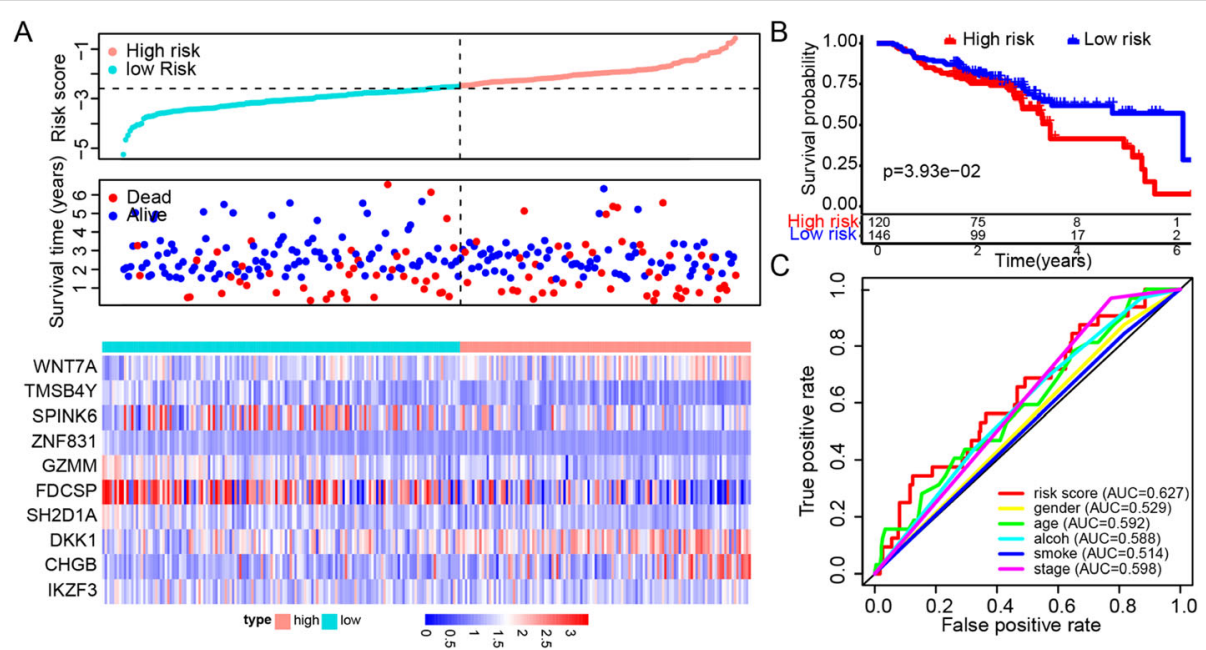

Fig. 3 Validation of the DEG-based gene signature prognostic model in GSE65858. A Risk score, vital status and heatmap of prognostic genes in the high- and low-risk groups. B Kaplan-Meier survival curves of the relative overall survival of high- and low-risk patients. C The ROC curve for one-year survival of the gene signature and clinical features 

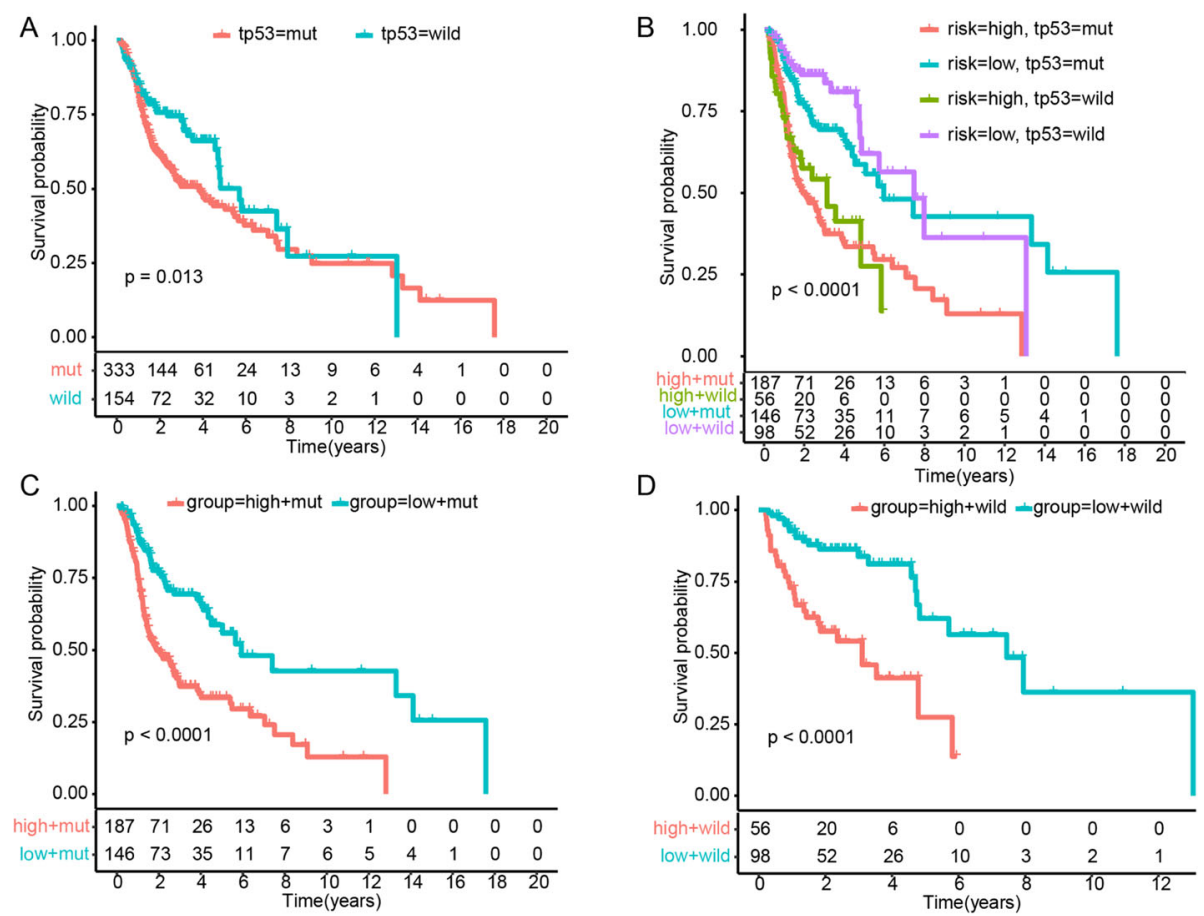

Fig. 4 Kaplan-Meier analysis of overall survival based on the combination of TP53 mutation status and risk score of the prognostic model. A TP53 mutation vs wildtype group. B TP53 mutation status with high-/low-risk group. C TP53 mutation subgroup with high-/low-risk group. D TP53 wildtype subgroup with high-/low-risk group

two sets based on the risk score were significantly distinct in the mutation and wildtype cohorts of HNSC, which indicated that the risk score was an independent predictor of prognosis $(P<0.0001$; Fig. $4 \mathrm{C}$ and $\mathrm{D})$.

\section{Immune cell infiltration landscapes of high- and low-risk patients with HNSC}

Immune infiltration of patients with HNSC between high- and low-risk groups was then elucidated. The ratios of 22 tumor-associated immune cell types were significantly distinct between high- and low-risk patients with HNSC (Fig. 5A). The correlation matrix indicated moderate correlations between immune cell rates (Fig. 5B). High-risk patients with HNSC tended to have higher rates of $\mathrm{CD} 4^{+}$resting memory $\mathrm{T}$ cells and $\mathrm{M0}$ / M2 macrophages and lower rates of naive $B$ cells, plasma cells, $\mathrm{CD}^{+} \mathrm{T}$ cells, activated memory $\mathrm{CD} 4^{+} \mathrm{T}$ cells, follicular helper $\mathrm{T}$ cells, regulatory $\mathrm{T}$ cells $(P<0.05$; Fig. $5 \mathrm{C}$ ). The difference of cells $\mathrm{CD} 4^{+}$resting memory $\mathrm{T}$ cells and M0/M2 macrophages $\mathrm{CD}^{+}{ }^{+} \mathrm{T}$ cells, activated memory $\mathrm{CD}^{+}{ }^{+} \mathrm{T}$ cells, follicular helper $\mathrm{T}$ cells between risk groups reappeared in GSE65858 cohort $(P<0.05$; Supplementary Figure 5) More details considering HPV status were recorded in Supplementary Table 2.

Antibodies that target immune checkpoints, such as CTLA-4 or PD-1, are the current strategies for cancer immunotherapy [32]. Many studies have indicated that
LAG3, LGALS9, HAVCR2 and TIGIT might be nextgeneration immune checkpoints [33-36]. We found that high-risk patients with HNSC expressed remarkably lower levels of CTLA4 $(P<0.01)$, LGALS9 $(P<0.05)$, LAG3 $(P<0.001), P D-1 \quad(P<0.0001)$, and TIGIT $(P<$ $0.0001)$, but higher levels of $P D-1 \quad(P<0.05$; Fig. $5 D)$. These results suggested that immunotherapy targeting immune checkpoints might be more effective for lowrisk patients.

\section{Immunotherapeutic and chemotherapeutic responses of high- and low-risk patients with HNSC}

Antibody targeting of immune checkpoint blockade provides a promising therapy for a variety of human cancers; however, partial and complete therapeutic resistance is commonly present in most patients. Therefore, we analyzed the clinical response to $P D-1$ and a group of chemical drugs in high- and low-risk patients with HNSC. Notably, anti-PD-1 therapy was less effective in most of the high-risk patients with $\operatorname{HNSC}(P<$ 0.01 ; Fig. $6 \mathrm{~A}$ and $\mathrm{B}$ ), which is consistent with the above hypothesis. The chemotherapeutic and targeted drugs used in the clinical practice, and clinical trials in HNSC showed differences in the predicted IC50 between highand low-risk patients with HNSC. Imatinib, Bortezomib, Bryostatin.1, Gemcitabine, Pazopanib, Sorafenib, and Tipifarnib significantly decreased the IC50 and increased 


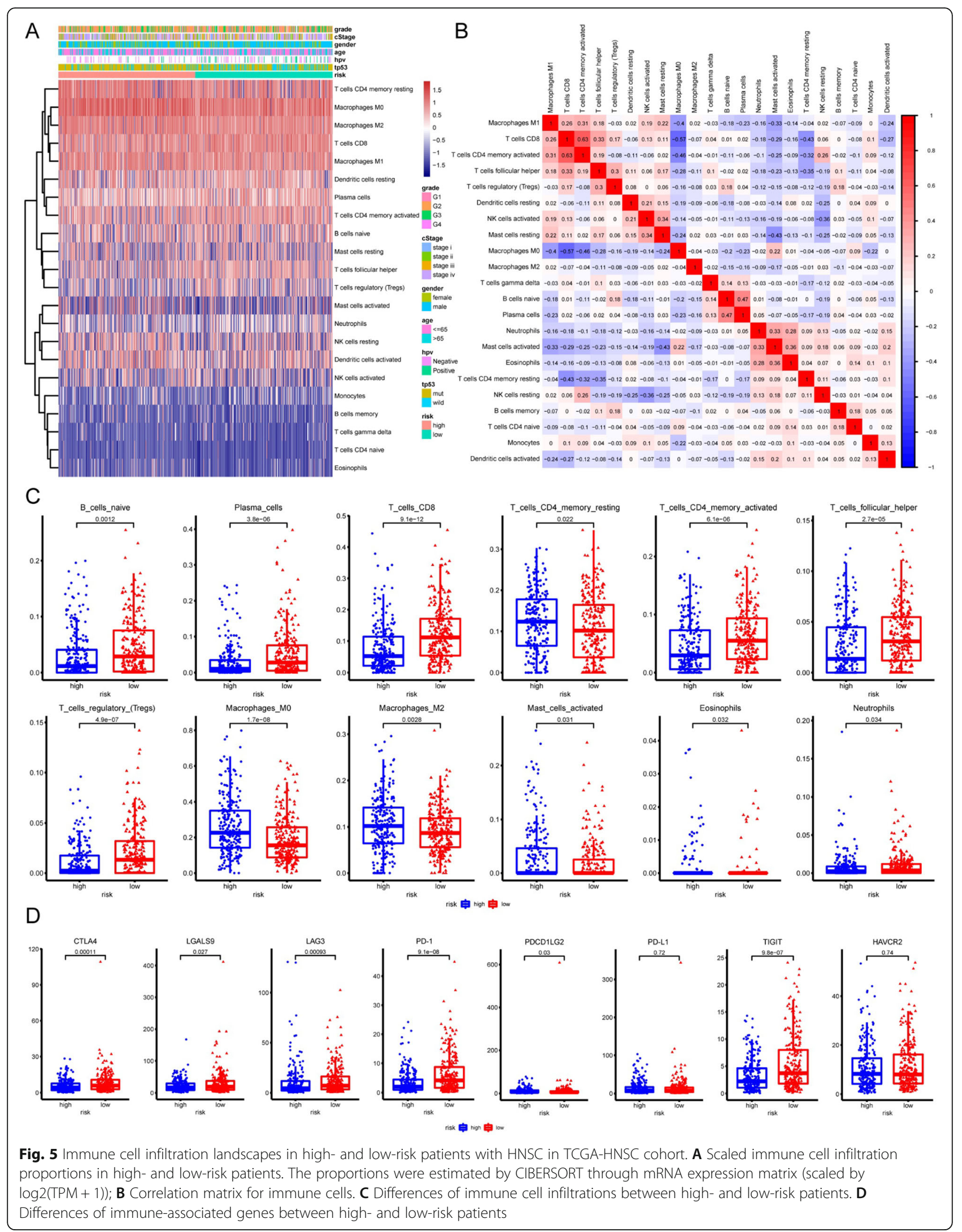




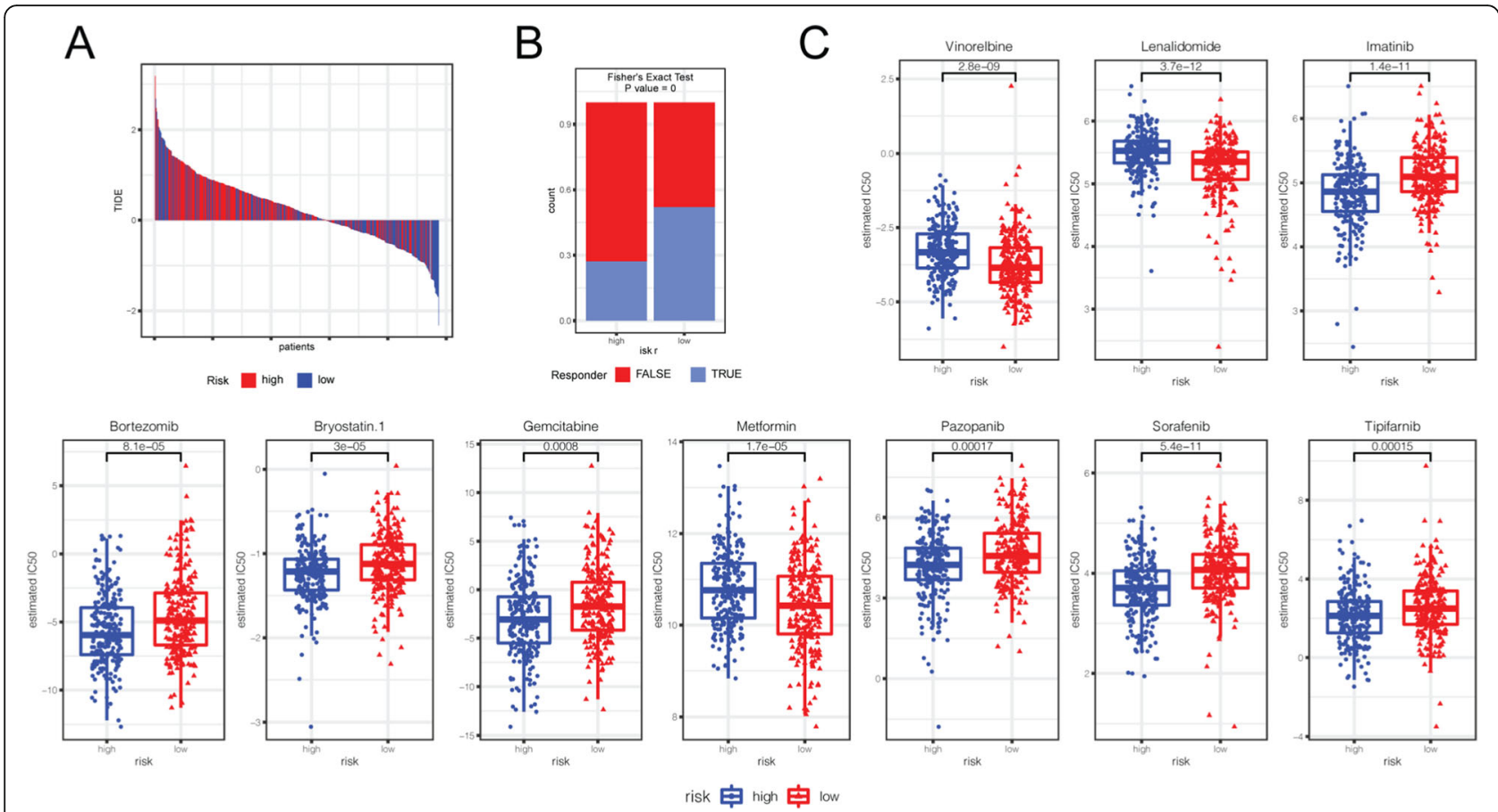

Fig. 6 Immunotherapeutic and chemotherapeutic responses in high- and low-risk patients with HNSC. A Immunotherapeutic responses to antiPD-1 therapy in high- and low-risk patients. B The P value is shown using Fisher's exact test to detect whether the immunotherapeutic response rates of the high-risk and low-risk groups were significantly different. C Drugs in clinics or in test for HNSC with significant differential chemotherapeutic responses $(P<0.001)$ in high- and low-risk patients predicted by the pRRopheic package in the $R$ language

the sensitivity for high-risk patients with $\operatorname{HNSC}(P<$ 0.001, Fig. 6C). Docetaxel, Erlotinib, Axitinib, Doxorubicin, Paclitaxel, and Rapamycin are also sensitive for high-risk patients with HNSC $(P<0.05$, Supplementary Figure 4). These results suggested that most chemotherapies might be more effective for high-risk patients.

\section{Correlations between the prognostic signature model and clinical characteristics}

To identify whether the risk score is an independent predictive factor in comparison with traditional clinical characteristics, univariate and multivariate Cox regression analyses were performed. In the HNSC cohort of TCGA, clinical stage and risk score were associated with poorer survival and could serve as specific predictors for prognostic assessment $(P<0.001$, Fig. $7 \mathrm{~A}$ and $\mathrm{B})$, as well as in the GSE65858 dataset $(P<0.01$, Fig. $7 D$ and $E)$. A prognostic nomogram based on multivariate analysis was used to further evaluate the prognostic effect. Compared with age and clinical stage, the risk score performed better at predicting the 1-, 2-, and 3-year overall survival (Fig. 7C and F).

\section{Discussion}

HNSC is the most common subtype of head and neck malignancies, with more than $40 \%$ mortality worldwide [37] and approximately $41 \%$ concordance with TP53 mutation [11]. Traditionally, clinicians plan treatment of HNSC based on clinical and pathological stage, status of HPV infection (particularly in OPSC), presence of a metastatic lymph node with or without extranodal extension (ENE), and depth of invasion (DOI) in oral cavity cancer [8]. In addition, the expression of molecular biomarkers can predict prognosis and monitor responses to therapies and even be therapeutic targets, such as epidermal growth factor (EGFR) monoclonal antibody (cetuximab) and immune checkpoint inhibitors targeting PD-1 [38]. Thus, the identification of novel targets or mutations is helpful for clinical strategies. An increasing number of studies have shown that the TP53 mutation is associated with HNSC prognosis [31] and acts as a predictor of the clinical response to HNSC treatment [39]. In our current study, we identified that TP53 was the most frequently mutated gene in $63 \%$ of $\mathrm{HPV}^{-}$ HNSC, the majority of which were missense mutations.

An increasing number of studies elsewhere have shown that TP53 plays a crucial role in tumor recognition by the immune system and antitumor immunosurveillance [40-43]. However, the bilateral functions of inhibiting $[42,44]$ or promoting $[45,46]$ antitumor immune activity indicate that the tumor immunity induced by TP53 mutations is attributed to tumor heterogeneity in different human cancers. According to the DEGs between the TP53-mutated and wildtype groups, GSEA 


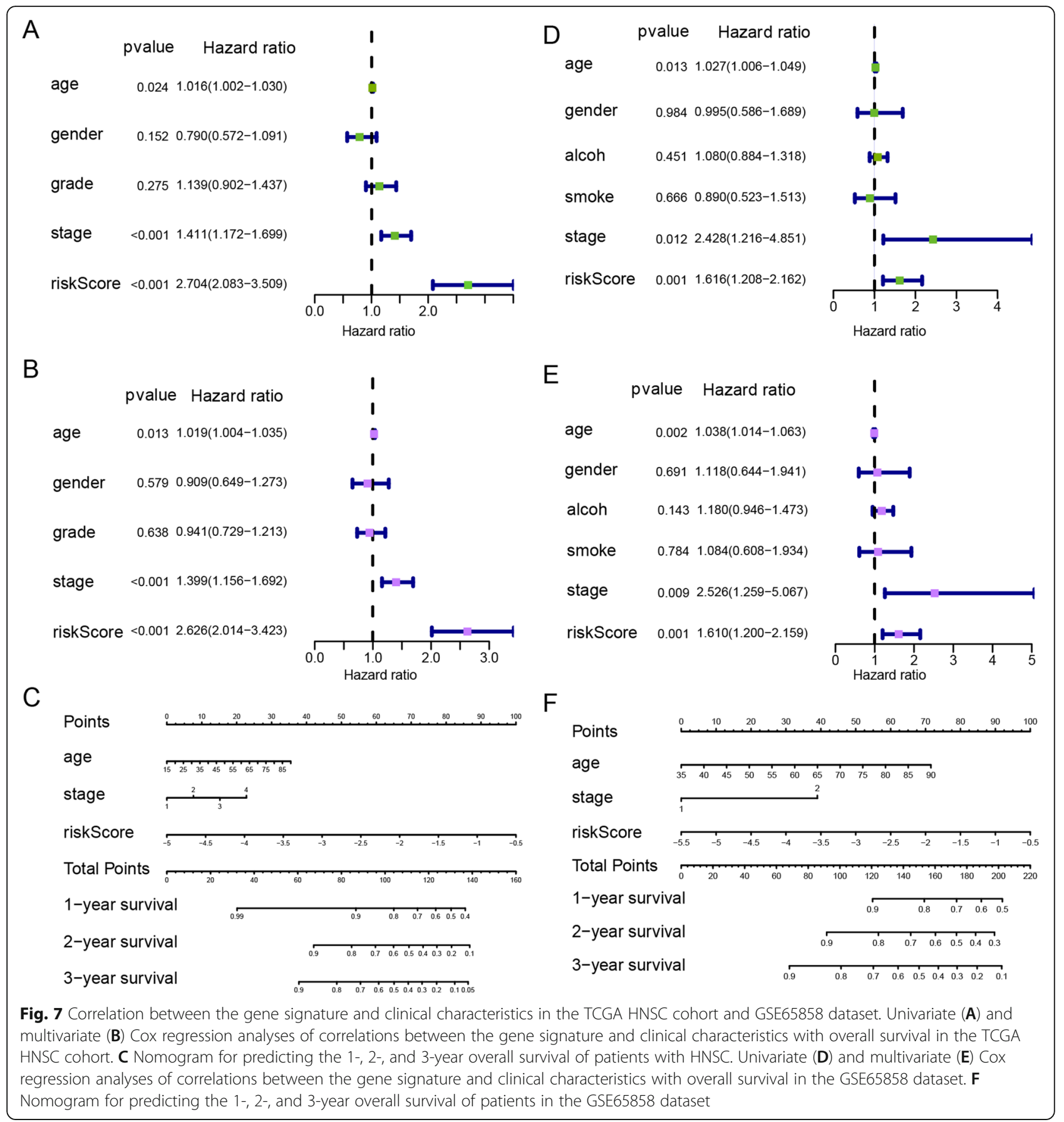

analysis was performed, and we found that TP53 mutations were associated with immune-related signaling pathways in patients with HNSC, including the T cell receptor signaling pathway, natural killer cell-mediated cytotoxicity, primary immunodeficiency, and antigen processing and presentation.

Given that the status of TP53 mutation functions as an effective prognostic predictor and therapeutic surveillance in HNSC [31], the risk score, a novel model based on ten genes affected by TP53, was first established to efficiently evaluate the overall survival likelihood of patients with HNSC. The results indicated that the risk score was more effective than clinical features, including age, sex, grade and clinical stage. The discovery cohort and the replication cohort are based on two different technologies (RNA-Seq vs Bead-array) which renders the comparison hazardous and could explain why the model does not perform so well in the validation cohort. $\mathrm{Pa}$ tients grouped as high-risk were more likely to have poorer overall survival. The risk score with TP53 
mutation exerted a more significant effect for the prediction of overall survival.

Immune cell infiltration is a significant characteristic of the tumor microenvironment (TME). The dual role of immunity between tumors and the host evolves the hypothesis of cancer immunosurveillance into cancer immunoediting, which is a dynamic process consisting of three phases: elimination, equilibrium, and escape [47]. The interferon gamma (INF) produced by infiltrated lymphocytes, such as natural killer cells and natural killer $\mathrm{T}$ cells, induces tumor death and secretion of several chemokines, which regulate the recruitment of more immune cells, such as how cytotoxic CD8+ T cells migrate to the tumor site and kill tumor cells with an immunogenic phenotype [48]. Tumor cell variants with genetic mutations or epigenetic changes acquire a nonimmunogenic phenotype and resistance to the immune system and then survive the elimination phase and enter the escape phase [47].

A growing amount of evidence shows that the presence of tumor-infiltrating lymphocytes (TILs) is associated with a favorable prognosis and a better response to therapies in a wide range of malignancies, including epithelial ovarian cancer, esophageal carcinoma, gastric carcinoma, colorectal cancer, lymph node melanoma, and primary cutaneous melanoma [49-54]. The prognostic role of TILs in HNSC is highly consistent with previous studies on other cancers. An immunohistochemical investigation showed that high $\mathrm{CD}^{+}$or $\mathrm{CD} 8^{+}$TILs in oral cancer were related to an improved prognosis [55], which was confirmed by a systematic review and metaanalysis [56]. A large retrospective study on HNSC also indicated that $\mathrm{CD}_{4}^{+}$and $\mathrm{CD} 8^{+} \mathrm{T}$ cells were independent factors for better overall survival and recurrence-free survival [57].

Loss of function or mutation of TP53 was perceived to be associated with inhibition or escape from antitumor immune responses [58]. Li $\mathrm{L}$ et al. reported that immune-stimulatory and immune-inhibitory signatures, CD8+/CD4+ regulatory $\mathrm{T}$ cells, pro-/anti-inflammatory cytokines, and M1/M2 macrophages were lower in TP53-mutated HNSC than in the wildtype subgroup [59]. Recently Huo M et al. established a prognostic risk model based on the TME score and found that patients in the high-risk group were more likely to have worse prognosis and a high frequency of TP53 mutation and expressed lower immune activation genes, CXCL9 and CXCL10, and immune checkpoint genes, IDO1 and HAVCR2 [60]. Consistently, our current study found that high-risk patients with HNSC generally had lower proportions of naive B cells, plasma cells, CD8+ T cells, activated memory CD4+ T cells, follicular helper $\mathrm{T}$ cells, and regulatory $\mathrm{T}$ cells. HNSC patients in the high-risk group expressed significantly lower levels of immune checkpoint genes, including PD-1, CTLA4, LGALS9, LAG3, and TIGIT.

$P D-1$ is an immune checkpoint that normally inhibits $\mathrm{T}$ cell activation, and tumor cells can suppress the antitumor immune response by upregulating the expression of PD-1 ligands [61]. Given that $P D-L 1$ is highly expressed in HNSC [62], inhibitors of $P D-1$ or $P D-L 1$ are thought to be a promising strategy to enhance $\mathrm{T}$ cell-mediated antitumor immunity and improve patient survival [63, 64]. In our current study, HNSC patients in the high-risk group tended to be unresponsive to antiPD-1 therapy. However, high-risk patients with HNSC were more sensitive to 28 chemotherapies. Collectively, the reason why high-risk patients with HNSC have poorer prognosis may be attributed to higher immunosuppression or lower immunoreactivity in the TME, which promotes tumor growth, metastasis, and resistance to therapies. These data suggested that the risk score system might be beneficial to individualized treatment for patients with HNSC.

Clinical stage and risk score significantly affected the overall survival of patients with HNSC, when compared with sex, grade, and alcohol and smoke consumption. Furthermore, the ten-gene risk score signature was associated with poorer survival and functioned as a predictor for prognostic assessment.

\section{Conclusion}

Our study suggested that the gene signature combined with the status of TP53 mutation offered a more effective method to evaluate prognosis and response to immunotherapies and chemotherapies for patients with HNSC.

\section{Supplementary Information}

The online version contains supplementary material available at https://doi. org/10.1186/s12885-021-08765-w.

Additional file 1: Supplementary Figure 1. DEGs and KM survival plot of patients with non-benign TP53 mutation and wildtype. (A) The volcano plot of DEGs between non-benign TP53 mutation and wildtype patient tissues. The DEG analysis were finished by using limma package. (B) The survival plot of HNSC patients classified by reannotated TP53 mutation status according SnpEFF IMPACT and PolyPhen annotation results, including benign (IMPACT was LOW and PolyPhen was benign), non-benign (other mutations: IMPACT was MODERATE/HIGH or PolyPhen was possibly_damaging/probably_damaging). (C) The survival plot of HNSC patients classified by PolyPhen annotated TP53 mutation status. The DEGs were identified by limma package (padj <0.05, $|\log F C|>1$ ).

Additional file 2: Supplementary Figure 2. The boxplot of ten prognostic genes expression in model of TCGA HNSC samples of TP53 mutation and wildtype. The $p$ values were calculated by Kruskal-Wallis test.

Additional file 3: Supplementary Figure 3. The KM survival plot TCGA HNSC patients stratified by HPV status and risk model. (A) HPV ${ }^{+}$vs $\mathrm{HPV}^{-}$group. (B) $\mathrm{HPV}^{+}$with high-/low-risk group. (C) $\mathrm{HPV}^{+}$subgroup with high-/low-risk group. (D) HPV subgroup with high-/low-risk group. 
Additional file 4: Supplementary Figure 4. Drugs in clinics or in test for HNSC without significant differential chemotherapeutic responses ( $p \geq 0.001$ ) in high- and low-risk patients. The results were predicted by the pRRopheic package in the R language, and the difference of IC50 were assessed with Kruskal-Wallis test.

Additional file 5: Supplementary Figure 5. Immune cell infiltration landscapes in high- and low-risk patients with HNSC in GSE65858 cohort. (A) Correlation matrix for immune cells.; (B) Scaled immune cell infiltration proportions in high- and low-risk patients (C) Differences of immune cell infiltrations between high- and low-risk patients. The difference of cell fractions were assessed with Kruskal-Wallis test.

Additional file 6: Supplemental Table 1. The immune and clinic characteristics between HPV negative patients and positive patients in TCGA HNSC. Briefly speaking HPV + HNSC has more infiltrated immune cells, especially tumor suppressive cells like memory B cell, CD8 T cells, activated memory CD4 T cells, T cells follicular helper, and less pro-tumor cells like Macrophages M2. Interestingly HPV + HNSC has a greater proportion of low risk group, and those HPV positive patients were younger (median age:57 vs 61) and of high pathological grade.

Additional file 7: Supplementary Table 2. The immune cell infiltration differences between patients of high risk and low risk stratified by HPV status. The immune cell proportion differences were mainly present in HPV-HNSC patient. Significant $p$ values were marked boldly.

Additional file 8: Supplementary Table 3. Univariate Cox analysis results of the 30 significant genes. Ten genes in risk model were marked boldly. Padj value were calculated by Bonferroni correction.

Additional file 9: Supplementary Table 4. Information of the ten genes in the prognostic model. Most of the ten genes were correlated with tumor or immune functions. Padj value and HR from coxph analysis and coefficients from risk model formula were displayed in the table.

\section{Acknowledgements}

We thank Min Sun, MD, PhD, Department of General Surgery, Taihe Hospital, Hubei University of Medicine, Shiyan, China, for review of this manuscript.

\section{Authors' contributions}

C. S. and P. G. designed the study. C. S. and S. L. carried out data acquisition and analysis. C. S. and P. G. wrote the manuscript. C. S. and X. T. contributed to preparing and making figures and Tables. X. W. and P. G. edited the manuscript. All authors have read and approved the final manuscript.

\section{Funding}

This study was sponsored by the Fund for Basic and Applied Basic Research at the Sichuan University West China Hospital of Stomatology (RD-02201914) for P.G.

\section{Availability of data and materials}

We downloaded VarScan 2-based somatic mutation data, RNA sequencing and clinical information data of the TCGA HNSC cohort from UCSC-Xena, dated August 28th, 2019. As a result, a total of 500 HNSC samples were enrolled in this analysis. The GSE65858 microarray dataset (https://www.ncbi. nlm.nih.gov/geo/query/acc.cgi?acc=GSE65858) comprises 270 HNSC specimens with gene expression profiles and their associated clinical characteristics [65]. All data were normalized in the R computing environment using the limma package. All analyses were conducted in accordance with relevant regulations and guidelines.

\section{Declarations}

\section{Ethics approval and consent to participate}

The original studies of TCGA and GEO database have obtained informed consents from the participants. All the experiment protocol for involving human data was in accordance with the Declaration of Helsinki and the protocol and approved by the Medical Ethics Committee of the West China Hospital of Stomatology, Sichuan University (WCHSIRB-OT-2020-069).

\section{Consent for publication}

Not applicable.

\section{Competing interests}

The authors declare that there is no conflict of interest.

\section{Author details}

${ }^{1}$ State Key Laboratory of Oral Diseases \& National Clinical Research Center for Oral Diseases \& Department of Head and Neck Oncology, West China Hospital of Stomatology, Sichuan University, NO.14, 3rd Section of Ren Min Nan Rd., Chengdu 610041, Sichuan, China. ${ }^{2}$ State Key Laboratory of Oral Diseases \& National Clinical Research Center for Oral Diseases \& Department of General and Emergency Dentistry, West China Hospital of Stomatology, Sichuan University, Chengdu, China.

Received: 9 May 2021 Accepted: 1 September 2021 Published online: 16 September 2021

\section{References}

1. Leemans CR, Braakhuis BJ, Brakenhoff RH. The molecular biology of head and neck cancer. Nat Rev Cancer. 2011;11(1):9-22. https://doi.org/10.1038/ nrc2982.

2. Yan K, Agrawal N, Gooi Z. Head and neck masses. Med Clin North Am. 2018; 102(6):1013-25. https://doi.org/10.1016/j.mcna.2018.06.012.

3. Global Burden of Disease Cancer C, Fitzmaurice C, Allen C, Barber RM, Barregard L, Bhutta ZA, et al. Global, regional, and national cancer incidence, mortality, years of life lost, years lived with disability, and disability-adjusted life-years for 32 cancer groups, 1990 to 2015: a systematic analysis for the global burden of disease study. JAMA Oncol. 2017;3(4):52448.

4. Miyauchi S, Kim SS, Pang J, Gold KA, Gutkind JS, Califano JA, et al. Immune modulation of head and neck squamous cell carcinoma and the tumor microenvironment by conventional therapeutics. Clin Cancer Res. 2019; 25(14):4211-23. https://doi.org/10.1158/1078-0432.CCR-18-0871.

5. Maier H, Dietz A, Gewelke U, Heller WD, Weidauer H. Tobacco and alcohol and the risk of head and neck cancer. Clin Investig. 1992;70(3-4):320-7. https://doi.org/10.1007/BF00184668.

6. Gillison ML, Chaturvedi AK, Anderson WF, Fakhry C. Epidemiology of human papillomavirus-positive head and neck squamous cell carcinoma. J Clin Oncol. 2015;33(29):3235-42. https://doi.org/10.1200/JCO.2015.61.6995.

7. Li H, Torabi SJ, Yarbrough WG, Mehra S, Osborn HA, Judson B. Association of human papillomavirus status at head and neck carcinoma subsites with overall survival. JAMA Otolaryngol Head Neck Surg. 2018;144(6):519-25. https://doi.org/10.1001/jamaoto.2018.0395.

8. Lydiatt WM, Patel SG, O'Sullivan B, Brandwein MS, Ridge JA, Migliacci JC, et al. Head and neck cancers-major changes in the American joint committee on cancer eighth edition cancer staging manual. CA Cancer J Clin. 2017;67(2):122-37. https://doi.org/10.3322/caac.21389.

9. Cramer JD, Burtness B, Le QT, Ferris RL. The changing therapeutic landscape of head and neck cancer. Nat Rev Clin Oncol. 2019;16(11):669-83. https:// doi.org/10.1038/s41571-019-0227-z.

10. Stransky N, Egloff AM, Tward AD, Kostic AD, Cibulskis K, Sivachenko A, et al. The mutational landscape of head and neck squamous cell carcinoma. Science. 2011;333(6046):1157-60. https://doi.org/10.1126/science.1208130.

11. Feldman R, Gatalica Z, Knezetic J, Reddy S, Nathan CA, Javadi N, et al. Molecular profiling of head and neck squamous cell carcinoma. Head Neck. 2016;38(Suppl 1):E1625-38. https://doi.org/10.1002/hed.24290.

12. Aubrey BJ, Strasser A, Kelly GL. Tumor-suppressor functions of the TP53 pathway. Cold Spring Harb Perspect Med. 2016;6(5). https://doi.org/10.1101/ cshperspect.a026062.

13. Lyu H, Li M, Jiang Z, Liu Z, Wang X. Correlate the TP53 mutation and the HRAS mutation with immune signatures in head and neck squamous cell cancer. Comput Struct Biotechnol J. 2019;17:1020-30. https://doi.org/10.101 6/j.csbj.2019.07.009.

14. Zhou G, Liu Z, Myers JN. TP53 mutations in head and neck squamous cell carcinoma and their impact on disease progression and treatment response. J Cell Biochem. 2016;117(12):2682-92. https://doi.org/10.1002/jcb.25592.

15. Ritchie ME, Phipson B, Wu D, Hu Y, Law CW, Shi W. Smyth GK: limma powers differential expression analyses for RNA-sequencing and microarray studies. Nucleic Acids Res. 2015;43(7):e47. https://doi.org/10.1093/nar/ gkv007.

16. Yu G, Wang LG, Han Y, He QY. clusterProfiler: an R package for comparing biological themes among gene clusters. Omics. 2012;16(5):284-7. https:// doi.org/10.1089/omi.2011.0118. 
17. Subramanian A, Tamayo P, Mootha VK, Mukherjee S, Ebert BL, Gillette MA, et al. Gene set enrichment analysis: a knowledge-based approach for interpreting genome-wide expression profiles. Proc Natl Acad Sci U S A. 2005;102(43):15545-50. https://doi.org/10.1073/pnas.0506580102.

18. Kanehisa M, Sato Y, Kawashima M, Furumichi M, Tanabe M. KEGG as a reference resource for gene and protein annotation. Nucleic Acids Res. 2016;44(D1):D457-62. https://doi.org/10.1093/nar/gkv1070.

19. Kanehisa M, Furumichi M, Tanabe M, Sato Y, Morishima K. KEGG: new perspectives on genomes, pathways, diseases and drugs. Nucleic Acids Res. 2017;45(D1):D353-d361. https://doi.org/10.1093/nar/gkw1092.

20. Meng S, Fan X, Zhang J, An R, Li S. GJA1 expression and its prognostic value in cervical cancer. Biomed Res Int. 2020;2020:8827920-10. https://doi. org/10.1155/2020/8827920.

21. Li B, Zhang B, Wang X, Zeng Z, Huang Z, Zhang L, et al. Expression signature, prognosis value, and immune characteristics of Siglec-15 identified by pan-cancer analysis. Oncoimmunology. 2020;9(1):1807291. https://doi.org/10.1080/2162402X.2020.1807291.

22. Zhu C, Wang X, Yang X, Sun J, Pan B, Zhang W, et al. Preoperative albuminbilirubin grade as a prognostic predictor in colorectal cancer patients who undergo radical resection. Cancer Manag Res. 2020;12:12363-74. https://doi. org/10.2147/CMAR.S285212.

23. Carron J, Costa APD, Rinck-Junior JA, Mariano FV, de Sá CB, Lima CSP, et al. Role of a genetic variation in the microRNA-4421 binding site of ERP29 regarding risk of oropharynx cancer and prognosis. Sci Rep. 2020;10(1): 17039. https://doi.org/10.1038/s41598-020-73675-Z.

24. Wang J, Wang Y, Xing P, Liu Q, Zhang C, Sui Y, et al. Development and validation of a hypoxia-related prognostic signature for breast cancer. Oncol Lett. 2020;20(2):1906-14. https://doi.org/10.3892/ol.2020.11733.

25. Liao X, Yang C, Huang R, Han C, Yu T, Huang K, et al. Identification of potential prognostic long non-coding RNA biomarkers for predicting survival in patients with hepatocellular carcinoma. Cell Physiol Biochem. 2018;48(5):1854-69. https://doi.org/10.1159/000492507.

26. Chen B, Khodadoust MS, Liu CL, Newman AM, Alizadeh AA. Profiling tumor infiltrating immune cells with CIBERSORT. Methods Mol Biol. 2018;1711:243-59.

27. Jiang P, Gu S, Pan D, Fu J, Sahu A, Hu X, et al. Signatures of T cell dysfunction and exclusion predict cancer immunotherapy response. Nat Med. 2018;24(10):1550-8. https://doi.org/10.1038/s41591-018-0136-1.

28. Geeleher P, Cox N, Huang RS. pRRophetic: an R package for prediction of clinical chemotherapeutic response from tumor gene expression levels. PLoS One. 2014;9(9):e107468. https://doi.org/10.1371/journal.pone.0107468.

29. Garnett MJ, Edelman EJ, Heidorn SJ, Greenman CD, Dastur A, Lau KW, et al. Systematic identification of genomic markers of drug sensitivity in cancer cells. Nature. 2012;483(7391):570-5. https://doi.org/10.1038/nature11005.

30. Balachandran VP, Gonen M, Smith JJ, DeMatteo RP. Nomograms in oncology: more than meets the eye. Lancet Oncol. 2015;16(4):e173-80. https://doi.org/10.1016/S1470-2045(14)71116-7.

31. Poeta ML, Manola J, Goldwasser MA, Forastiere A, Benoit N, Califano JA, et al. TP53 mutations and survival in squamous-cell carcinoma of the head and neck. N Engl J Med. 2007;357(25):2552-61. https://doi.org/10.1056/ NEJMoa073770.

32. Ribas A, Wolchok JD. Cancer immunotherapy using checkpoint blockade. Science. 2018;359(6382):1350-5. https://doi.org/10.1126/science.aar4060.

33. Andrews LP, Marciscano AE, Drake CG, Vignali DA. LAG3 (CD223) as a cancer immunotherapy target. Immunol Rev. 2017;276(1):80-96. https://doi. org/10.1111/imr.12519.

34. Chauvin JM, Zarour HM. TIGIT in cancer immunotherapy. J Immunother Cancer. 2020;8(2). https://doi.org/10.1136/jitc-2020-000957.

35. Daley D, Mani VR, Mohan N, Akkad N, Ochi A, Heindel DW, et al. Dectin 1 activation on macrophages by galectin 9 promotes pancreatic carcinoma and peritumoral immune tolerance. Nat Med. 2017;23(5):556-67. https://doi. org/10.1038/nm.4314.

36. Das M, Zhu C, Kuchroo VK. Tim-3 and its role in regulating anti-tumor immunity. Immunol Rev. 2017;276(1):97-111. https://doi.org/10.1111/imr.12 520.

37. Leemans CR, Snijders PJF, Brakenhoff RH. The molecular landscape of head and neck cancer. Nat Rev Cancer. 2018;18(5):269-82. https://doi.org/10.1038/ nrc.2018.11.

38. Gougis P, Moreau Bachelard C, Kamal M, Gan HK, Borcoman E, Torossian N, et al. Clinical development of molecular targeted therapy in head and neck squamous cell carcinoma. JNCI Cancer Spectr. 2019;3(4):pkz055.
39. Perrone F, Bossi P, Cortelazzi B, Locati L, Quattrone P, Pierotti MA, et al. TP53 mutations and pathologic complete response to neoadjuvant cisplatin and fluorouracil chemotherapy in resected oral cavity squamous cell carcinoma. J Clin Oncol. 2010;28(5):761-6. https://doi.org/10.1200/JCO.2009.22.4170.

40. Dong ZY, Zhong WZ, Zhang XC, Su J, Xie Z, Liu SY, et al. Potential predictive value of TP53 and KRAS mutation status for response to PD-1 blockade immunotherapy in lung adenocarcinoma. Clin Cancer Res. 2017; 23(12):3012-24. https://doi.org/10.1158/1078-0432.CCR-16-2554.

41. Guo G, Yu M, Xiao W, Celis E, Cui Y. Local activation of p53 in the tumor microenvironment overcomes immune suppression and enhances antitumor immunity. Cancer Res. 2017;77(9):2292-305. https://doi.org/10.11 58/0008-5472.CAN-16-2832.

42. Jiang Z, Liu Z, Li M, Chen C, Wang X. Immunogenomics analysis reveals that TP53 mutations inhibit tumor immunity in gastric cancer. Transl Oncol. 2018;11(5):1171-87. https://doi.org/10.1016/j.tranon.2018.07.012.

43. Zitvogel L, Kroemer G. CANCER. A p53-regulated immune checkpoint relevant to cancer. Science. 2015;349(6247):476-7. https://doi.org/10.1126/ science.aac8475

44. Xiao W, Du N, Huang T, Guo J, Mo X, Yuan T, et al. TP53 mutation as potential negative predictor for response of anti-CTLA-4 therapy in metastatic melanoma. EBioMedicine. 2018;32:119-24. https://doi.org/10.101 6/j.ebiom.2018.05.019.

45. Ham SW, Jeon HY, Jin X, Kim EJ, Kim JK, Shin YJ, et al. TP53 gain-of-function mutation promotes inflammation in glioblastoma. Cell Death Differ. 2019; 26(3):409-25. https://doi.org/10.1038/s41418-018-0126-3.

46. Liu Z, Jiang Z, Gao Y, Wang L, Chen C, Wang X. TP53 mutations promote immunogenic activity in breast cancer. J Oncol. 2019;2019:5952836-19. https://doi.org/10.1155/2019/5952836.

47. Dunn GP, Old LJ, Schreiber RD. The three Es of cancer immunoediting. Annu Rev Immunol. 2004;22(1):329-60. https://doi.org/10.1146/annurev. immunol.22.012703.104803.

48. O'Donnell JS, Teng MWL, Smyth MJ. Cancer immunoediting and resistance to T cell-based immunotherapy. Nat Rev Clin Oncol. 2019;16(3):151-67. https://doi.org/10.1038/s41571-018-0142-8.

49. Zhang L, Conejo-Garcia JR, Katsaros D, Gimotty PA, Massobrio M, Regnani G, et al. Intratumoral T cells, recurrence, and survival in epithelial ovarian cancer. N Engl J Med. 2003;348(3):203-13. https://doi.org/10.1056/NEJMoa020177.

50. Schumacher K, Haensch W, Röefzaad C, Schlag PM. Prognostic significance of activated CD8(+) T cell infiltrations within esophageal carcinomas. Cancer Res. 2001;61(10):3932-6.

51. Ishigami S, Natsugoe S, Tokuda K, Nakajo A, Che X, Iwashige H, et al. Prognostic value of intratumoral natural killer cells in gastric carcinoma. Cancer. 2000;88(3):577-83. https://doi.org/10.1002/(SICI)1097-0142(20000201 88:3<577::AID-CNCR13>3.0.CO;2-V.

52. Naito Y, Saito K, Shiiba K, Ohuchi A, Saigenji K, Nagura H, et al. CD8+ T cells infiltrated within cancer cell nests as a prognostic factor in human colorectal cancer. Cancer Res. 1998;58(16):3491-4.

53. Mihm MC Jr, Clemente CG, Cascinelli N. Tumor infiltrating lymphocytes in lymph node melanoma metastases: a histopathologic prognostic indicator and an expression of local immune response. Lab Investig. 1996;74(1):43-7.

54. Clemente CG, Mihm MC Jr, Bufalino R, Zurrida S, Collini P, Cascinelli N. Prognostic value of tumor infiltrating lymphocytes in the vertical growth phase of primary cutaneous melanoma. Cancer. 1996;77(7):1303-10. https://doi.org/1 0.1002/(SICI)1097-0142(19960401)77:7<1303::AID-CNCR12>3.0.CO;2-5.

55. Wolf GT, Chepeha DB, Bellile E, Nguyen A, Thomas D, McHugh J. Tumor infiltrating lymphocytes (TIL) and prognosis in oral cavity squamous carcinoma: a preliminary study. Oral Oncol. 2015;51(1):90-5. https://doi.org/1 0.1016/j.oraloncology.2014.09.006.

56. de Ruiter EJ, Ooft ML, Devriese LA, Willems SM. The prognostic role of tumor infiltrating T-lymphocytes in squamous cell carcinoma of the head and neck: a systematic review and meta-analysis. Oncoimmunology. 2017; 6(11):e1356148. https://doi.org/10.1080/2162402X.2017.1356148.

57. Nguyen N, Bellile E, Thomas D, McHugh J, Rozek L, Virani S, et al. Tumor infiltrating lymphocytes and survival in patients with head and neck squamous cell carcinoma. Head Neck. 2016;38(7):1074-84. https://doi.org/1 0.1002/hed.24406.

58. Blagih J, Buck MD, Vousden KH. p53, cancer and the immune response. J Cell Sci. 2020;133(5). https://doi.org/10.1242/jcs.237453.

59. Li L, Li M, Wang X. Cancer type-dependent correlations between TP53 mutations and antitumor immunity. DNA Repair (Amst). 2020:88:102785. https://doi.org/10.1016/j.dnarep.2020.102785. 
60. Huo M, Zhang Y, Chen Z, Zhang S, Bao Y, Li T. Tumor microenvironment characterization in head and neck cancer identifies prognostic and immunotherapeutically relevant gene signatures. Sci Rep. 2020;10(1):11163. https://doi.org/10.1038/s41598-020-68074-3.

61. Syn NL, Teng MWL, Mok TSK, Soo RA. De-novo and acquired resistance to immune checkpoint targeting. Lancet Oncol. 2017;18(12):e731-41. https:// doi.org/10.1016/S1470-2045(17)30607-1.

62. Zandberg DP, Strome SE. The role of the PD-L1:PD-1 pathway in squamous cell carcinoma of the head and neck. Oral Oncol. 2014;50(7):627-32. https:// doi.org/10.1016/j.oraloncology.2014.04.003.

63. Kiyota N, Hasegawa Y, Takahashi S, Yokota T, Yen CJ, Iwae S, et al. A randomized, open-label, phase III clinical trial of nivolumab vs. therapy of investigator's choice in recurrent squamous cell carcinoma of the head and neck: a subanalysis of Asian patients versus the global population in checkmate 141. Oral Oncol. 2017;73:138-46. https://doi.org/10.1016/j.ora loncology.2017.07.023.

64. Harrington KJ, Ferris RL, Blumenschein G Jr, Colevas AD, Fayette J, Licitra L, et al. Nivolumab versus standard, single-agent therapy of investigator's choice in recurrent or metastatic squamous cell carcinoma of the head and neck (CheckMate 141): health-related quality-of-life results from a randomised, phase 3 trial. Lancet Oncol. 2017;18(8):1104-15. https://doi. org/10.1016/S1470-2045(17)30421-7.

65. Wichmann G, Rosolowski M, Krohn K, Kreuz M, Boehm A, Reiche A, et al. The role of HPV RNA transcription, immune response-related gene expression and disruptive TP53 mutations in diagnostic and prognostic profiling of head and neck cancer. Int J Cancer. 2015;137(12):2846-57. https://doi.org/10.1002/ijc.29649.

\section{Publisher's Note}

Springer Nature remains neutral with regard to jurisdictional claims in published maps and institutional affiliations.

Ready to submit your research? Choose BMC and benefit from:

- fast, convenient online submission

- thorough peer review by experienced researchers in your field

- rapid publication on acceptance

- support for research data, including large and complex data types

- gold Open Access which fosters wider collaboration and increased citations

- maximum visibility for your research: over $100 \mathrm{M}$ website views per year

At $\mathrm{BMC}$, research is always in progress.

Learn more biomedcentral.com/submissions 